\title{
NEW ONSET DIABETES AFTER TRANSPLANTATION IN RENAL TRANSPLANT RECIPIENTS AT TYGERBERG HOSPITAL
}

\author{
Nabeel A. Bapoo, Johan D. Nel
}

Division of Nephrology, Department of Internal Medicine, Tygerberg Hospital and The University of Stellenbosch, Cape Town, South Africa

\begin{abstract}
New Onset Diabetes after Transplantation (NODAT) is a common complication of renal transplantation worldwide. However, there is very little data available regarding this condition in Sub-Saharan Africa. This study was therefore conducted in order to determine the incidence of NODAT and its associated risk factors in a South African renal transplant population. A retrospective analysis was conducted on 221 patients who underwent renal transplants at Tygerberg Hospital during the period January 1st 1995 to December 31st 2008.

Specific information was retrieved from patient files in order to determine the study outcomes. Fifty of the 221 patients were found to have NODAT reflecting an incidence of $22.6 \%$ (95\% CI 0.17-0.28). The cumulative incidences of NODAT at three, 12 and 36 months were $9 \%, 12 \%$ and $17 \%$ respectively. The mean time to onset of NODAT was 18 months after transplantation (95\% CI 11.3-25.3) and the mean age at diagnoses of NODAT was 41 years (95\% CI 38.9-44.8). Transplantation at the age of 40 years (odds ratio $=1.05$ ) as well the usage of tacrolimus (odds ratio $=0.43$ ) was found to increase the risk of developing NODAT. The development of NODAT did not have any effect on graft or patient survival in this study.
\end{abstract}

The incidence of NODAT in a South African population appears to be as high as it is worldwide with the first year posttransplantation carrying the greatest risk for its development. The risk for development of NODAT is increased as the time post-transplantation increases. Transplantation from the age of 40 years and the use of tacrolimus appear to be the most significant risk factors.

\section{BACKGROUND}

The annual incidence of end-stage renal disease varies from 100 per million population in the United Kingdom to 336 per million population in the United States [1]. The incidence of chronic kidney disease in Sub-Saharan Africa is estimated to be three to four times higher than in more developed countries [2]. It is a well-established fact that renal transplantation is the best form of renal replacement therapy. Renal transplant recipients have a longer life expectancy than patients who receive any form of dialysis ( 8.17 years versus 4.32 years) and are less likely to develop cardiovascular and cerebrovascular disease [3] - this reference reflects elderly patients. Transplantation also improves the quality of life (QOL) in large groups of patients when compared to those on dialysis [4].

Complications commonly occur after renal transplantation. Amongst those is New Onset Diabetes after Transplantation
(NODAT). Studies have shown an association with the increased risk of cardiovascular disease in transplant recipients which ultimately is a major cause of myocardial infarction and death in renal transplant recipients [5-7]. NODAT reduces both graft and patient survival and is also an independent predictor of death-censored graft failure [810].

The incidence of NODAT increases as the time after transplantation increases [4]. Its cumulative incidences at three, 12 and 36 months post-renal transplantation is about $9 \%, 12 \%$ and $24 \%$ respectively $[11,12]$. Incidence of NODAT varies amongst population groups and various institutions worldwide. In the United States two studies at different centres showed similar figures. The incidence ranged from $9.1 \%$ to $24 \%$ versus $5.9 \%$ to $29.8 \%$ (the variation was due to different incidence rates at different periods of time) [11, 12]. In a UK study the incidence ranged from $15 \%$ to $20 \%$ [11]. The incidences are very similar in most studies [11-17].

Address Correspondence to: Dr Nabeel A. Bapoo, Division of Nephrology, Department of Internal Medicine, Tygerberg Hospital and The University of Stellenbosch, Cape Town, South Africa. 
The consequence of NODAT is an increase in the costs required for management of this already expensive group of patients. In the United States the costs of treating transplant recipients who develop NODAT is estimated to be $\$ 12,000$ more (per patient) than for patients without diabetes in the first year post transplant and $\$ 20,000$ more by the end of the second year post transplant $[18,19]$. There is very little variation in costs when comparing patients using different forms of immunosuppressive therapy [18]. More important is the effect on patient and graft survival. Graft survival is reduced due to the development of diabetic nephropathy and hypertension and patient survival is reduced due to an increased incidence of opportunistic infections and cardiovascular disease $[19,20]$. Patient survival has also been shown to be significantly reduced. In a study by Demirci et al, the 10-year mortality of patients with NODAT was nearly twice that of non-diabetic transplant recipients [14]. It is not surprising that quality of life in this unique group of patients is adversely affected [21].

Not all patients develop NODAT but many studies have identified risk factors associated with the development of this condition. These risk factors include: African American ethnicity, body mass index (BMI) more than $30 \mathrm{~kg} / \mathrm{m}^{2}$, age at transplantation, family history of diabetes, impaired glucose tolerance pre-transplant, metabolic syndrome, recipient of deceased donor kidneys, immunosuppressive therapy including corticosteroids and tacrolimus, Hepatitis $\mathrm{C}$ virus infection, and autosomal dominant polycystic kidney disease [11-17, 19, 22-23].

Data on NODAT in Sub-Saharan Africa is limited. In the only Southern African retrospective study done at Johannesburg General Hospital by Harrichund et al, the incidence of NODAT was found to be $15.6 \%$ with a mean time to onset of 22.9 months [24]. The highest incidence was found to be amongst Black patients with the use of tacrolimus as an immunosuppressive agent [24]. These findings fall in line with worldwide trends.

The aims of this study were therefore to determine the incidence of NODAT amongst renal transplant recipient patients at Tygerberg Hospital, whether the patient population with NODAT fit the risk profile described in the literature, and to examine the effect of NODAT on the time to graft failure or death.

\section{METHODS}

Files of patients who had renal transplants during the period January 1st 1995 to December 31st 2008 were retrieved for a retrospective analysis. Patients who received renal transplants at Tygerberg Hospital during this period were identified and included in the study. Those who had diabetes mellitus prior to transplantation and those transferred to other hospitals for follow up were excluded.

Files were obtained from the Renal Transplant Clinic (living patients), the Renal Unit's Archives, and the Tygerberg Hospital Medical Records department. The following information was extracted from the folders: presence or absence of NODAT, age at transplant, weight at transplant, plasma glucose at transplant, gender, race, time from transplant to diagnoses of NODAT, number of extra steroid pulses, calcineurin inhibitor used, type of transplant, number of transplants, weight at diagnoses of NODAT, age at diagnoses of NODAT, time to graft failure, and time to death.

In this study the diagnosis of NODAT was based on the current World Health Organization criteria for diabetes mellitus (25):

Fasting plasma glucose $\geq 7.0 \mathrm{mmol} / 1(126 \mathrm{mg} / \mathrm{dl})$ or 2-hour plasma glucose $\geq 11.1 \mathrm{mmol} / 1(200 \mathrm{mg} / \mathrm{dl})$

The primary objective of the study to describe the incidence of NODAT in the Tygerberg patient population was presented by means of proportions and appropriate 95\% confidence intervals for proportions. The secondary objective to determine whether the patients fit the risk profile described in previous literature was presented by means of the student's t-test and the Mann-Whitney $U$ test. A chi-squared analysis was used to determine any association between the presence or absence of a risk factor and NODAT. Odds ratios were calculated after univariate and multivariate logistic regression analysis of possible risk factors.

\section{RESULTS}

Three hundred and forty-seven patients had renal transplants during the study time period. Of these, only 245 patient files were found. Twenty-four patients were excluded from the study. Seventeen had diabetes mellitus prior to transplantation and seven were transferred to other hospitals for follow up. The eventual study cohort consisted of 221 patients (Figure 1).

The gender distribution was 106 males and 115 females. In terms of race, 152 were Coloured (mixed race), 33 Black, 31 White and five Asian. One hundred and eighty-seven patients received cadaveric transplants, 33 received living related transplants and one patient received a living unrelated transplant. Calcineurin inhibitors used were cyclosporine in 173 patients, tacrolimus in 42 patients and sirolimus in five patients. One hundred and ninety-one patients received a total of one transplant, 23 received two transplants and six received three transplants. Baseline characteristics are summarised in Table 1.

Fifty of the 221 patients were found to have NODAT reflecting an incidence of $22.6 \%$ (95\% CI 0.17-0.28) (Figure 2). The cumulative incidences of NODAT at three, 12 and 36 months were $9 \%, 12 \%$ and $17 \%$ respectively (Figure 2). The mean time to onset of NODAT was 18 months (95\% CI 11.3-25.3) after transplantation and the mean age at diagnoses of NODAT was 41 years (95\% CI 38.9-44.8) (Figure 3). A mean increase in weight from the time of transplantation to the diagnoses of NODAT of $7.7 \mathrm{~kg}(95 \%$ CI 2.8-12.6) was also found in the NODAT group. 
In the group who developed NODAT, 25 patients were male and 25 female. Of these, 34 patients were Coloured, eight White, seven Black and one Asian. Calcineurin inhibitors used in the NODAT group were cyclosporine in 32 patients, tacrolimus in 15 patients and sirolimus in three patients. Forty-one patients received cadaveric renal transplants while only nine received living related transplants. These findings have been summarised in Table 2 .

The NODAT group of patients was compared to the nonNODAT group in order elicit any significant differences in risk factors and long term outcome. The student's t-test was used to compare the mean age at transplant and mean weight at transplant between the two groups. In terms of age a statistically significant difference was found between the two groups. The mean age at transplant in the NODAT group was 40 years while in the non-NODAT group it was $37(p=0.03)$ (Figure 4). There was also a difference in the mean weight at transplant between the two groups but this was not statistically significant.

The Mann-Whitney $U$ test was used to compare the average number of steroid pulses, average number of transplants, average time to graft failure as well as average time to death between the groups but none of these showed any statistical significance.

Chi-squared tests were used to identify risk factors for NODAT which were then evaluated by means of multivariate logistic regression analysis. Results of this analysis showed that the following risk factors proved to be statistically significant: average age at transplant, average weight at transplant and the type of calcineurin inhibitor used. These risk factors were entered into a multivariate model and odds ratios were calculated. The results showed that an average age at transplant of 40 years (odds ratio $=1.05$ ), as well the usage of tacrolimus (odds ratio $=0.43$ ) increases the risk of developing NODAT. The average weight at transplant of $70 \mathrm{~kg}$ as a risk factor for NODAT was found not to be statistically significant (odds ratio $=1.0$ ).

\section{DISCUSSION}

In this study the incidence of NODAT at Tygerberg Hospital was found to be $22.6 \%$ with cumulative incidences at three, 12 and 36 months of $9 \%, 12 \%$ and $17 \%$ respectively. This is in keeping with incidences which have been reported in previous studies from the USA, UK, Asia as well as Southern Africa [11-16, 26]. The mean time to onset of NODAT was 18 months. This is similar to a previous Southern African study but does not fall in line with other previous worldwide studies which have shown median times to onset of NODAT to be much earlier $[8,13-15,17,24,26]$. This may be due to differences in the populations represented in the various studies. It must be noted that despite showing a mean onset of 18 months, most patients (50\%) developed NODAT within the first 10 months after transplantation - and this may be in keeping with other studies (Figure 3).

A mean age at transplantation of 40 years was found to be a significant risk factor for the development of NODAT compared to patients who did not develop NODAT having a mean age of 37 . The difference in age at transplantation between the two groups was not as great as described by Harrichund et al but still is significant [24]. Many previous studies also described age as a significant risk factor for the development of NODAT [11, 12, 16, 17, 19, 26]. The mean age at transplantation of 40 years, combined with the fact that half of the patients developed NODAT within one year after transplantation, probably explains why the average age at diagnoses of NODAT in this study was 41 years. It must be noted that patients who are accepted for renal replacement at Tygerberg Hospital are significantly younger than patients in the private sector or in other first world settings, and this is due to stringent selection criteria and limited financial resources.

This study also found that the use of calcineurin inhibitors, specifically tacrolimus (when compared to cyclosporine) was a significant risk factor for the development of NODAT. This falls in line with most previous studies worldwide [12$14,19,24,27,28]$. A meta-analysis comparing the effects of tacrolimus and cyclosporine was done by Webster et al [29]. In this systematic review tacrolimus was favoured over cyclosporine as it showed improved survival rates, decreased incidences of acute rejection and lower mean creatinine levels at six months post-transplantation [29]. No difference was found between the two drugs in the incidence of opportunistic infections or malignancies. Both drugs had various side effects but as expected the meta-analysis showed an increased risk of NODAT with tacrolimus.

In a study comparing cost effectiveness between the two drugs in the United Kingdom, tacrolimus was found to be the more cost effective option even though the long term costs were higher due to its superior overall survival, survival with a functioning graft and rejection-free survival [30]. Evidence is emerging that despite its costs and possible adverse effects, tacrolimus is the favoured drug as its benefits by far outweigh its risks.

At Tygerberg Hospital, patients are started on cyclosporine in addition to prednisone and azathioprine as primary immunosuppression after renal transplantation. Patients are changed to tacrolimus only if they experience severe adverse effects due to cyclosporine or if they develop steroidresistant acute rejection. It is for this reason that only $19 \%$ of the study patients received the drug. However, evidence is emerging worldwide for tacrolimus to be used as a firstline immunosuppressant and this practice may eventually be implemented in South Africa.

The addition of extra steroid pulses for treatment of acute rejection was not found to be a risk factor for the development of NODAT unlike the findings of other studies $[10,26]$.

Black people have been identified as a risk for NODAT in most previous studies $[11,12,19,24,28]$. The same was not found in this study possibly due to the fact that the patient population was not as racially diverse as in other studies or that the genetic predisposition of South African Blacks to the development of diabetes mellitus is different to Black patients in other first world countries. Harrichund et al 
showed similar results [24]. The fact that the number of Black patients in this study were so small $(15 \%)$ is another reason for them not being identified as a significant risk factor for the development of NODAT. It is noteworthy that eight $(53 \%)$ of the 15 Black patients who received renal transplants at Tygerberg Hospital developed NODAT. A future study which includes more Black renal transplant patients may expose this risk factor as significant for the development of NODAT in a South African population.

There was no variation with gender for the development of NODAT. The study population consisted of an equal number of males and females and this was also reflected amongst those who developed NODAT (Tables 1, 2). Gender was therefore not considered a risk factor for the development of NODAT. Other studies have also shown the same results [13-16].

This study did not show that recipients of cadaver kidneys were more at risk for NODAT than patients who received living related transplants as shown in other studies [11, 12, 19, 24, 28]. This may be due to the relatively small number of living related transplants amongst the study population $(15 \%)$.

Several studies have also shown that body mass indexes of more than $30 \mathrm{~kg} / \mathrm{m}^{2}$ as well as a family history of diabetes mellitus are independent risk factors for NODAT [11, 12, $15,16,23,24]$. This study was inconclusive in terms of these risk factors as patient heights pre transplantation were never recorded and therefore it was not possible to calculate the body mass index for each patient. Most patient records were also not clear in terms of family history of diabetes. This study however shows a clear association between NODAT and an increase in weight of $7.7 \mathrm{~kg}$ from the time of transplantation to the diagnoses thereof.

There have been studies looking at the pre-existing renal disease as a risk factor for the development of NODAT. Specifically, autosomal dominant polycystic kidney disease (ADPKD) has been found to be a risk factor for its development $[22,23]$. Our study was unable to determine any association between the two conditions as there were limited numbers of patients (only 10 patients in the study had ADPKD) with this disease included in the study.

The influence of dialysis modality prior to transplantation has also been studied recently. Courivard et al determined that the dialysis modality pre transplantation had no influence on the development of post-transplant diabetes mellitus [31, 32]. In contrast, a study by Madziarska et al found that peritoneal dialysis appears to be a possible novel risk factor which may increase the risk of NODAT development [33]. At Tygerberg Hospital the majority of patients are started on peritoneal dialysis and are then transferred to haemodialysis within an average time of 18 months due to failure of the peritoneal membrane (personal communication, Dr R Adeniyi, Tygerberg Hospital Renal Unit). Most patients therefore underwent both forms of dialysis before transplantation which made it difficult to draw any conclusions regarding the impact of peritoneal dialysis on the development of NODAT in our transplant population.
Hepatitis $\mathrm{C}$ virus infection has been shown to be an independent risk factor for the development of NODAT in many studies $[11-14,16,19]$. In one study the prevalence of Hepatitis $\mathrm{C}$ as a risk factor for NODAT was particularly high in patients using tacrolimus [14]. However the exact reason for this association is not presently understood. During the study period no transplants were performed on Hepatitis C positive patients and it could therefore not be examined as a risk factor for NODAT.

The effect of NODAT on graft and patient survival was examined in this study as previous studies have shown that it reduces both [8-11, 26, 34]. There was no difference found between time to graft failure or time to death between patients with NODAT and those without. Therefore in this population no clear effect was shown in terms of graft and patient survival. This again is similar to what was shown by Harrichund et al [24].

Recent studies with regards to NODAT have looked at the existence of genetic polymorphisms in renal transplant recipients, some of which are thought to be protective against NODAT and others which have been implicated as causative factors for its development. Plasminogen activator inhibitor-1 5G/5G genotype homozygosity has been associated with a significantly lower rate of development of NODAT and is thought to be protective [35]. Dutkiewicz et al found that a polymorphism of the GPX1 gene may be associated with the development of NODAT in Polish subjects [36]. In Korea, studies have implicated various genetic polymorphisms as risk factors for the development of NODAT. These include: CCL5, TCF7L2, SLC30A8, HHEX, CDKAL1 and many others [37-39]. Many other studies are looking at genetic polymorphisms in an attempt to better understand the disease process. Due to financial constraints and the retrospective nature of this study the role of genetic polymorphisms was not examined.

\section{CONCLUSION}

The incidence of NODAT in a South African population appears to be as high as it is worldwide with the first year post-transplantation carrying the greatest risk for its development. The risk for development of NODAT is increased as the time post-transplantation increases. An age of 40 years or older at transplantation and the use of tacrolimus appear to be the most significant risk factors. An increase in weight of $7.7 \mathrm{~kg}$ post-transplantation may be associated with the development of NODAT. This study did not identify race, gender or cadaveric transplants as risk factors for the development of NODAT. NODAT was not shown to reduce graft failure or patient survival.

The findings of this study closely reflect what was found in the only other South African study looking at NODAT by Harrichund et al [24]. Similar incidences and risk factors were found in that study. However, similarities are also seen with many of the first world NODAT studies and this may reflect the lifestyle changes in the South African population which may have lead to the increased development of 
diabetes mellitus amongst all patients including those who underwent renal transplantation.

The roles of genetic polymorphisms as risk or protective factors are becoming more evident with more research being done in this field. These polymorphisms may eventually be used as potential treatment targets for the prevention or treatment of NODAT in the future. However, more research in this field is required.

\section{STUDY LIMITATIONS}

As this was a retrospective study from patient records, the data retrieved was not always complete. For example, body mass index could not be calculated as heights were not done on all patients' pre-transplantation and family history of diabetes mellitus was not always recorded in the notes. Hepatitis $\mathrm{C}$ as a risk factor for NODAT could not be evaluated in this study. A number of files were missing resulting in the patient cohort being smaller than expected. However, despite the relatively high dropout rate the results obtained were still of statistical significance.

\section{RECOMMENDATIONS}

Pre-transplant screening of patients in terms of family history of diabetes mellitus, body mass index, impaired glucose tolerance and patient age should be mandatory in all patients. This would aid in identifying patients who are at risk to develop NODAT. More intensive screening and appropriate management can then be instituted at an early stage. Post-transplantation patients should be monitored strictly in terms of fasting plasma glucose weekly for the first four weeks post-transplantation, then every three months for one year and annually thereafter [40]. Patients who have developed NODAT should be treated according to normal guidelines for diabetes mellitus.

Aspirin has been recommended in these patients at a dosage of $65-100 \mathrm{mg} /$ day for primary prevention for cardiovascular disease [40]. Aspirin therapy has been found to be beneficial after myocardial infarction even in patients who have chronic kidney disease and may have uremic platelet dysfunction [41]. It has also been associated with improved allograft function and survival [41]. However, it is important to note that the cardio protective effect of aspirin is limited mainly by the risk for serious gastrointestinal bleeding, a risk that may be higher in renal transplant recipients [41]. Therefore aspirin should be used with caution and at a low dosage.

A prospective study to address the shortfalls of this study is recommended, to provide a more detailed evaluation of this condition.

Any comments on early use of basal insulin and potential place of new oral diabetic drugs like Vildagliptin?

\section{ADDENDUM: FIGURES AND TABLES}

Figure 1: Patient selection

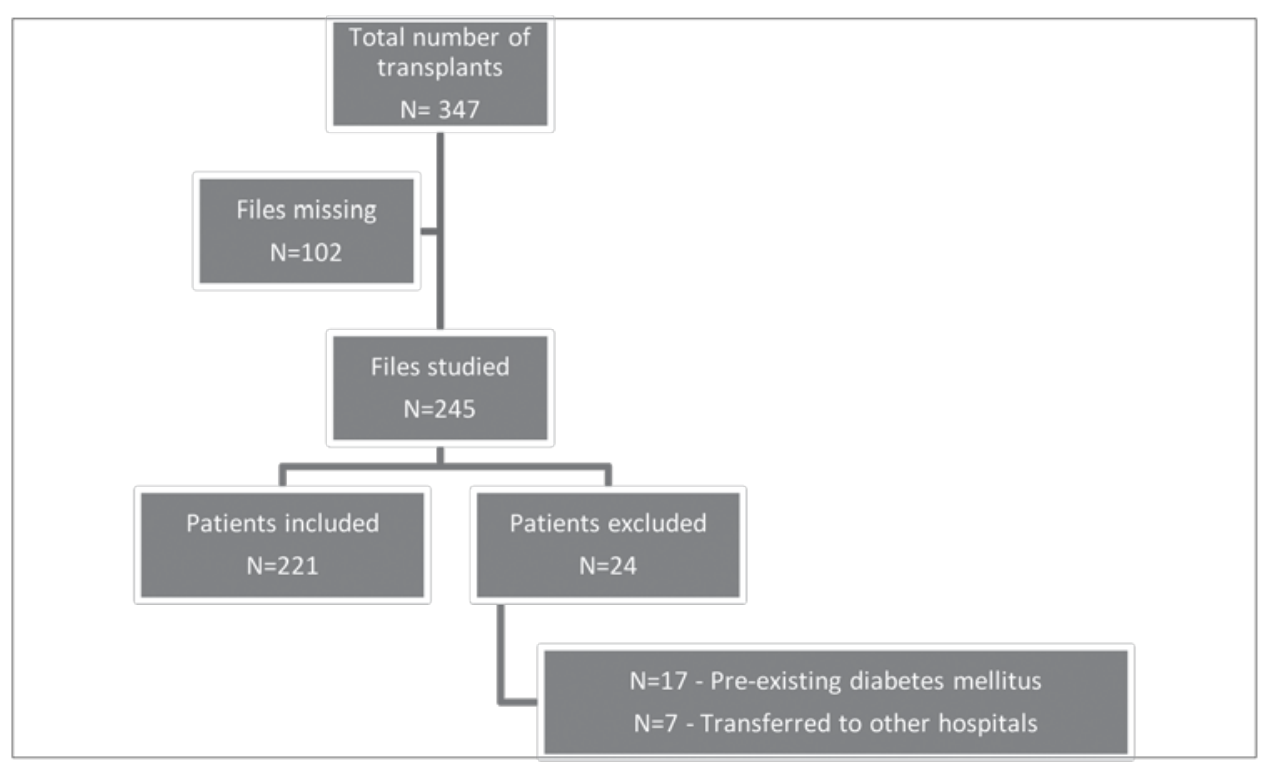


Figure 2: Incidence of NODAT

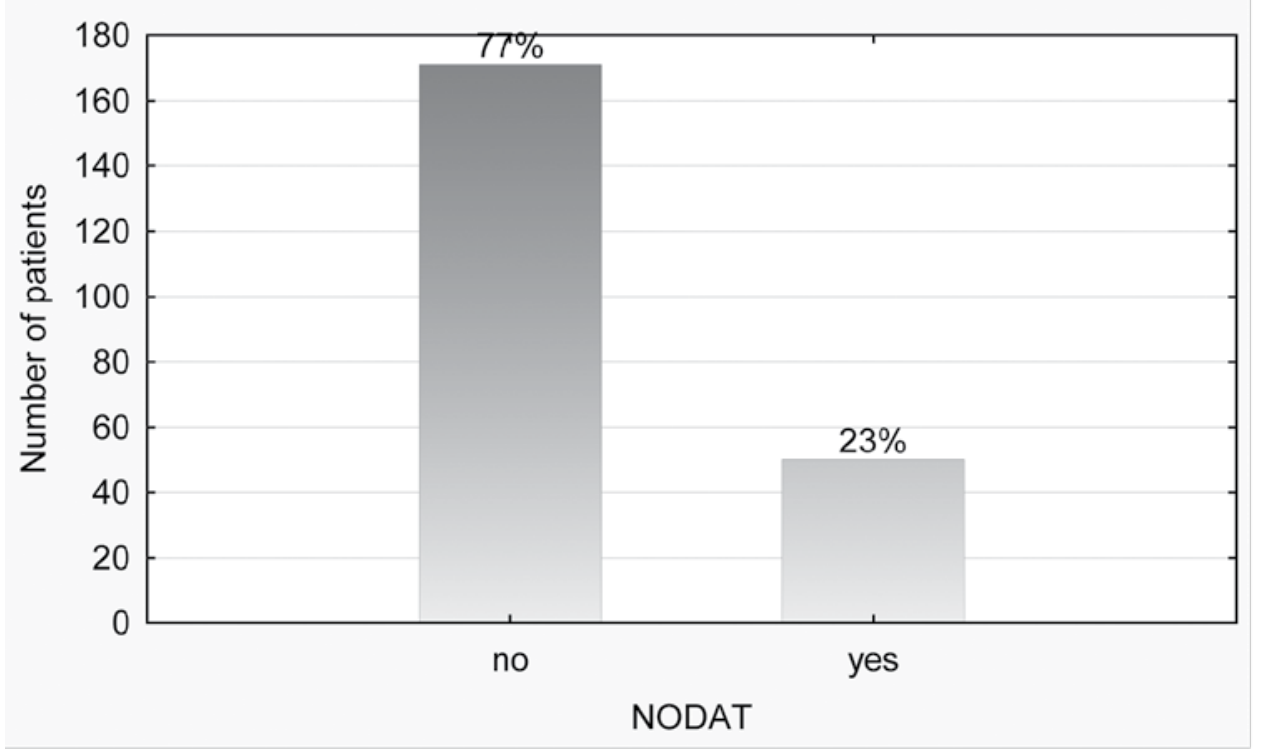

Figure 2 shows an incidence of NODAT of $23 \%$ in the Tygerberg population.

Figure 3: Time from transplant to development of NODAT

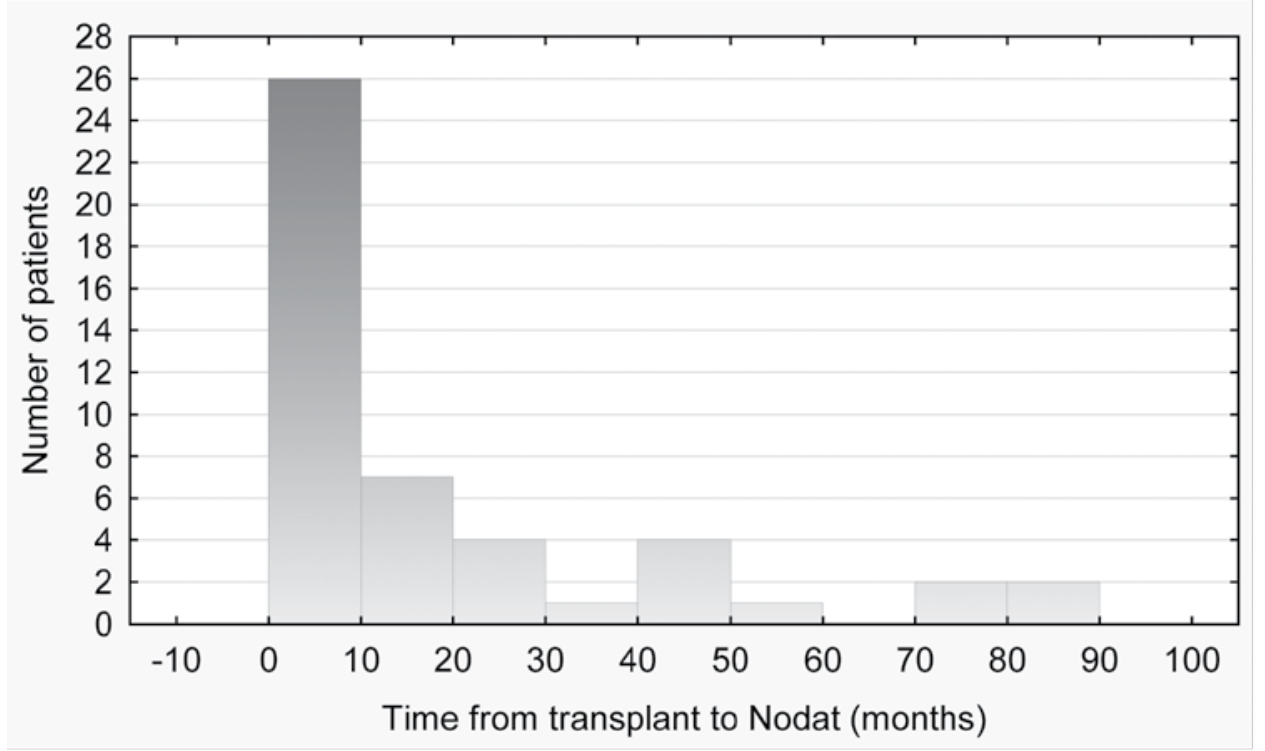

Figure 3 shows that although the mean time to onset of NODAT was 18 months, most patients developed the condition within the first year post-transplantation. 
Figure 4: Age at transplant ( $p=0.03)$ (Student's $t$-test)

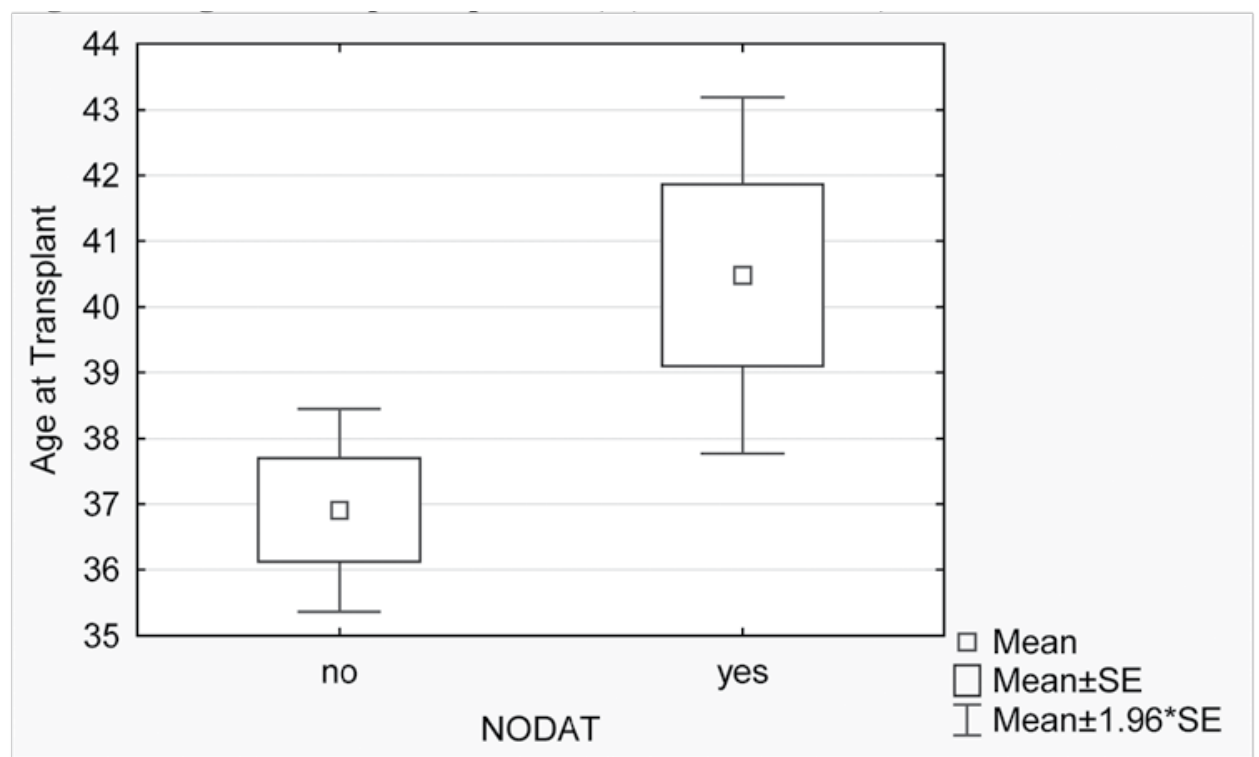

Figure 4 shows a statistically significant difference in the mean age at transplant between patients with and without NODAT - 40 years of age in the NODAT group versus 37 years in the Non-NODAT group.

Table 1: Baseline characteristics

\begin{tabular}{|ll|}
\hline Variable & $\begin{array}{l}\text { Number of } \\
\text { patients }\end{array}$ \\
Gender & \\
Male & \\
Female & $106(48 \%)$ \\
& $115(52 \%)$ \\
Race & \\
Coloured & \\
Black & $152(69 \%)$ \\
White & $33(15 \%)$ \\
Asian & $31(14 \%)$ \\
Calcineurin Inhibitor & $5(2 \%)$ \\
Cyclosporine & \\
Tacrolimus & $173(78 \%)$ \\
Sirolimus & $42(19 \%)$ \\
& $5(2 \%)$ \\
Type of transplant & \\
Cadaver & \\
Living related & $187(85 \%)$ \\
Living unrelated & $33(15 \%)$ \\
Number of & $1(0 \%)$ \\
transplants & \\
1 & \\
2 & $191(86 \%)$ \\
3 & $23(10 \%)$ \\
& $6(3 \%)$ \\
& \\
\hline
\end{tabular}


Table 2: Patient characteristics: NODAT vs Non-NODAT

\begin{tabular}{|lll|}
\hline Variable & $\begin{array}{l}\text { NODAT } \\
\text { patients }\end{array}$ & $\begin{array}{l}\text { Non-NODAT } \\
\text { patients }\end{array}$ \\
\hline Gender & & \\
Male & & \\
Female & $25(50 \%)$ & $81(47 \%)$ \\
Race & $25(50 \%)$ & $90(53 \%)$ \\
Coloured & & \\
Black & $34(68 \%)$ & $118(69 \%)$ \\
White & $8(16 \%)$ & $25(15 \%)$ \\
Asian & $7(14 \%)$ & $24(14 \%)$ \\
& $1(2 \%)$ & $4(2 \%)$ \\
Calcineurin Inhibitor & & \\
Cyclosporine & $32(64 \%)$ & $141(82 \%)$ \\
Tacrolimus & $14(30 \%)$ & $28(16 \%)$ \\
Sirolimus & $3(6 \%)$ & $2(1 \%)$ \\
Type of transplant & & \\
Cadaver & $41(82 \%)$ & $146(86 \%)$ \\
Living related & $9(18 \%)$ & $24(14 \%)$ \\
& & \\
\hline
\end{tabular}

\section{REFERENCES}

1. Hamer RA, El Nahas AM, The Burden Of Chronic Kidney Disease, British Medical Journal, 2006, Mar 11, 332(7541):563-564.

2. Naicker S, End-Stage Renal Disease In Sub-Saharan Africa, Ethnicity \& Disease, 2009, Spring, 19 (1 Suppl 1):S1-13-5.

3. Gabriel C, Oniscu, Brown H, John L, Forsythe R, How Great Is The Survival Advantage Of Transplantation Over Dialyses In Elderly Patients?, Nephrology Dialysis Transplantation, 2004, 19:945-951.

4. Valderrabano F, Jofre R, Lopez-Gomez JM, Quality Of Life In End-Stage Renal Disease Patients, American Journal of Kidney Diseases, 2001, Sep 38(3):443464.

5. Lindholm A, Albrechtsen D, Frodin L, Tufveson G, Persson NH, Lundgren G, Ischemic Heart Disease: Major Cause Of Death And Graft Loss After Renal Transplantation In Scandinavia, Transplantation, 1995, 60(5).

6. Ducloux D, Kazory A, Chalopin J, Post-Transplant Diabetes Mellitus And Atherosclerotic Events In Renal Transplant Recipients: A Prospective Study, Transplantation, 2005, 79(4):438.
7. Lentine KL, Brennan DC, Schnitzler MA, Incidence And Predictors Of Myocardial Infarction After Kidney Transplantation, 2005, 16(2):496.

8. Joss N, Staatz CE, Thomson AH, Jardine AG, Predictors Of New Onset Diabetes After Renal Transplantation, Clinical Transplantation, 2007, JanFeb, 21(1):136-143.

9. Balla A, Chobanian M, New Onset Diabetes After Transplantation: A Review Of Recent Literature, Current Opinion in Organ Transplant, 2009, Aug, 14(4):375379.

10. Rodrigo E, Fernandez-Fresnedo G, Valero R, Ruiz JC, Pinera C, Palomar R, et al, New Onset Diabetes After Kidney Transplantation: Risk Factors, Journal of the American Society of Nephrology, 2006, Dec, 17(12 Suppl 3):S291-5.

11. Cosio FG, Pesavento TE, Osei K, Henry ML, Ferguson RM, Post-Transplant Diabetes Mellitus: Increasing Incidence In Renal Allograft Recipients Transplanted In Recent Years, Kidney International, 2001, Feb, 59(2):732-737.

12. Kasiske BL, Snyder JJ, Gilbertson D, Matas AJ, Diabetes Mellitus After Kidney Transplantation In The United States, American Journal of Transplantation, 2003, Feb, 3(2):178-185. 
13. Madhav D, Ram R, Dakshinamurty KV, PostTransplant Diabetes Mellitus: Analysis Of Risk Factors, Effects On Biochemical Parameters And Graft Function Five Years After Renal Transplantation, Transplant Proceedings, 2010, 42(10):4069.

14. Demirci MS, Toz H, Yilmaz F, Ertilav M, Asci G, Ozkahya M, Zeytinoglu A, Nart D, Ok E, Risk Factors And Consequences Of Post-Transplant Diabetes Mellitus, Clinical Transplantation, 2010, 24(5):E170.

15. Allamani M, Sennesael J, Vendemeulenbroucke, Post-Transplantation Diabetes Mellitus: A Long-Term Retrospective Cohort Study, Transplant Proceedings, 2010, 42:4378.

16. Ali IH, Adberrahim E, Ben Abdelghani K, Barbouch S, Mchirgui N, Khiari K, Chérif M, Ounissi M, Ben Romhane N, Ben Abdallah N, Ben Abdallah T, Ben Maiz H, Khedher A, Incidence And Risk Factors For Post-Renal Transplant Diabetes Mellitus, Transplant Proceedings, 2011, 43(2):568.

17. Bora GS, Guleria S, Reddy VS, Tandon N, Gupta N, Gupta S, Bhowmik D, Risk. Factors For The Development Of New Onset Diabetes Mellitus In A Living Related Renal Transplant Program, Transplant Proceedings, 2010, 42(10):4072.

18. Woodward RS, Schnitzler MA, Baty J, Lowell JA, Lopez-Rocafort L, Haider S, Woodworth TG, Brennan DC, Incidence And Cost Of New Onset Diabetes Mellitus Among US Wait-Listed And Transplanted Renalallograft Recipients, American Journal of Transplantation, 2003, (3):590-598.

19. AJ Krentz DW, New Onset Diabetes After Transplantation, 2006, 23(1):34-42.

20. Cosio FG, Pesavento TE, Kim S, Osei K, Henry M, Ferguson RM, Patient Survival After Renal Transplantation:IV. Impact Of Post-Transplant Diabetes, Kidney International, 2002, 62:1440-1446.

21. Chakkera HA, Knowler WC, Devarapalli Y, Weil EJ, Heilman RL, Dueck, Mulligan DC, Reddy KS, Moss AA, Mekeel KL, Mazur MJ, Hamawi K, Castro JC, Cook CB, Relationship Between Inpatient Hyperglycemia And Insulin Treatment After Kidney Transplantation And Future New Onset Diabetes Mellitus, Clinical Journal of the American Society of Nephrology 5, 2010, 5:1669-1675.

22. Hamer RA, Chow CL, Ong AC, McKane WS, Polycystic Kidney Disease Is A Risk. Factor For New Onset Diabetes After Transplantation, Transplantation, 2007, (83):1-36.

23. De Mattos AM, Olyaei AJ, Prather JC, Golconda MS, Barry JM, Norman DJ, Autosomal-Dominant Polycystic Kidney Disease As A Risk Factor For Diabetes Mellitus
Following Renal Transplantation, Kidney International 2007, 67(2):712.

24. Harrichund P, Naicker S, Raal F, Becker P, New Onset Diabetes Mellitus After Renal Transplantation, Journal of Endocrinology, Metabolism and Diabetes of South Africa, 2008, 13(3):98.

25. World Health Organization/International Diabetes Federation, Definition And Diagnosis Of Diabetes Mellitus And Intermediate Hyperglycaemia. 2006, available at: http://www.who.int/diabetes/ publications/Definition $\% 20$ and $\% 20$ diagnosis $\% 20$ of $\% 20$ diabetes_new.pdf. Accessed September/08, 2009.

26. Yong Mong Bee,1MBBS (S'pore), MRCP, Hong Chang Tan,1MBBS (S'pore), MRCP, M Med (Int Med), Tunn Lin Tay,1MBBS (S'pore), MRCP, Terence YS Kee,2BMBS (Flinders), MRCP,SuYen Goh,1MBBS (S'pore), MRCP, Peng Chin Kek,1MBBS (S'pore), MRCP, Incidence And Risk Factors For Development Of New Onset Diabetes After Kidney Transplantation, Annals Academy of Medicine Singapore, 2011, 40:160-67, 2011, 40(4):160.

27. Coresh J, Astor BC, Greene T, Eknoyan G, Levey AS, Prevalence Of Chronic Kidney Disease And Decreased Kidney Function In The Adult US Population: Third National Health And Nutrition Examination Survey, American Journal of Kidney Diseases, 2003, Jan, 41(1):1-12.

28. Pham PT, Pham PC, Lipshutz GS, Wilkinson AH, New Onset Diabetes Mellitus After Solid Organ Transplantation, Endocrinology and Metabolism Clinics of North America, 2007, Dec, 36(4):873-90; vii.

29. Webster AC, Woodroffe RC, Taylor RS, Chapman JR, Craig JC, Tacrolimus Versus Ciclosporin As Primary Immunosuppression For Kidney Transplant Recipients: Meta-Analysis And Meta-Regression Of Randomised Trial Data, British Medical Journal, doi:10 1136/bmj 38569471007 AE.

30. Orme ME, Jurewicz WA, Kumar N, McKechnie TL, The Cost Effectiveness Of Tacrolimus Versus Microemulsified Cyclosprin. A 10-Year Model Of Renal Transplant Outcomes, Pharmacoeconomics, 2003, 21 (17):1263-1276.

31. Courivaud C, Potential Influence Of Dialysis Modality On Post-Transplantation Diabetes Mellitus Risk, Nephrology Dialysis Transplantation,2010, 26(6):2063.

32. Courivaud $\mathrm{C}$, Ladrière $\mathrm{M}$, Toupance $\mathrm{O}$, Caillard $\mathrm{S}$, de Ligny BH, Ryckelynck J-P, Moulin B, Rieu P, Frimat L, Chalopin J-M, Chauvé S, Kazory A, Ducloux D, Impact Of Pre-Transplant Dialysis Modality On PostTransplant Diabetes Mellitus After Kidney Transplantation, 
Clinical Transplantation, 2011, (25):794.

33. Madziarska K, Weyde W, Krajewska M, Patrzalek D, Janczak D, Kusztal M, Augustyniak-Bartosik H, Szyber P, Kozyra C, Klinger M, The Increased Risk Of Post-Transplant Diabetes Mellitus In Peritoneal DialysisTreated Kidney Allograft Recipients, Nephrology Dialysis Transplantation, 2011, Apr, 26(4):1396-401.

34. Kasiske BL, Snyder JJ, Gilbertson D, Matas AJ, Diabetes Mellitus After Kidney Transplantation In The United States, American Journal of Transplantation, 2003, Feb, 3(2):178-185.

35. Horng-Rong Chang, Shun Fa Yang, Jen-Pi Tsai, Ming-Chia Hsieh, Sheng-Wen Wu, Hui-Ching Tsai, Tung-Wei Hung, Jun-Huang Huang, Jong-Da Lian Plasminogen Activator Inbibitor-1 5G/5G Genotype Is A Protective Factor Preventing Post-Transplant Diabetes Mellitus, Clinica Chimica Acta, 2011, 412:322.

36. Dutkiewicz G, Domanski L, Pawlik A, BinczakKuleta A, Safranow K, Ciechanowicz A, Dziedziejko V, Pietrzak-Nowacka M, Ciechanowski K, Polymorphisms of Superoxide Dismutase, Glutathione Peroxidase And Catalase Genes In Patients With PostTransplant Diabetes Mellitus, Archives of Medical Research, 2010,41(5):350.

37. Eun Seok Kang, Myoung Soo Kim, Yu Seun Kim, Kyu Yeon Hur, Seung Jin Han, Chang Mo Nam, Chul Woo Ahn, Bong Soo Cha, Soon Il Kim, Hyun
Chul Lee, A Variant Of The Transcription Factor 7-Like 2 (TCF7L2) Gene And The Risk Of Post-Transplantation Diabetes Mellitus In Renal Allograft Recipients, Diabetes Care, 2008, 31(1):63.

38. Eun Seok Kang, Myoung Soo Kim, Chul Hoon Kim, Chung Mo Nam, Seung Jin Han, Kyu Yeon Hur, Chul Woo Ahn, Bong Soo Cha, Soon Il Kim, Hyun Chul Lee, Yu Seun Kim, Association of Common Type 2 Diabetes Risk Gene Variants And Post-Transplantation Diabetes Mellitus In Renal Allograft Recipients In Korea, Transplantation, 2009, 88(5):693.

39. Jeong KH, Moon JY, Chung JH, Kim YH, Lee TW, Significant Associations Between CCL5 Gene Polymorphisms And Post-Transplantational Diabetes Mellitus In Korean Renal Allograft Recipients, American Journal of Nephrology, 2010, 32(4):368.

40. Kasiske BL, Zeier MG, Chapman JR, Craig JC, Ekberg H, Garvey CA, Green MD, Jha V, Josephson MA, Kiberd BA, Kreis HA, McDonald RA, Newmann MA, Obrador GT, Vincenti FG, Cheung M, Earley A, Gowri Raman, Abariga S, Wagner M, Balk EM, KDIGO Clinical Practice Guideline For The Care Of Kidney Transplant Recipients: A Summary, International Society of Nephrology, 2009.

41. Shirali AC, Bia MJ, Management Of Cardiovascular Disease In Renal Transplant Recipients, Clinical Journal of American Society of Nephrology, 2008, 3(2):491. 